\title{
Absence of localization in a disordered one-dimensional ring threaded by an Aharonov-Bohm flux
}

\author{
Jean Heinrich:* \\ Département de Physique, B5a, Université de Liège, \\ Sart Tilman, B-4000 Liège, Belgium
}

(Dated: November 20, 2018)

\begin{abstract}
Absence of localization is demonstrated analytically to leading order in weak disorder in a onedimensional Anderson model of a ring threaded by an Aharonov-Bohm (A-B) flux. The result follows from adapting an earlier perturbation treatment of disorder in a superconducting ring subjected to an imaginary vector potential proportional to a depinning field for flux lines bound to random columnar defects parallel to the axis of the ring. The absence of localization in the ring threaded by an A-B flux for sufficiently weak disorder is compatible with large free electron type persistent current obtained in recent studies of the above model.
\end{abstract}

PACS numbers: 72.15.Rn,73.23.Ra, 73.63.Nm

*Electronic address: J.Heinrichs@ulg.ac.be 
The nature (localized or delocalized) of the eigentstates in a disordered metallic ring threaded by an Aharonov-Bohm (A-B) flux is expected to strongly influence the magnitude of the persistent current[1] which may be induced in the ring. In recent years the existence of persistent current has been demonstrated experimentally in metallic and semiconducting mesoscopic ring systems [2, 3, 4, 5, 6]. The various theoretical predictions of the magnitude of persistent current are, however, stagnating around values lying between one- and two orders of magnitude below the experimental results[7].

While in a disordered linear chain (and, likewise, in an infinite disordered ring as well) all eigenstates are localized [8, 9], the precise effect of an A-B flux on these states in a ring has, to out knowledge, not been discussed in detail so far. The aim of this note is to analyse the problem, using a simple extension of earlier work [10, 11] on localization in a one-dimensional ring in the presence of a non-hermitian field, in the context of depinning of flux lines bound to columnar defects in a superconductor[12].

The one-dimensional disordered ring threaded by an A-B flux $\phi$ is modelled by a tight binding system composed of $N$ one-orbital atomic sites, $n=1,2, \ldots, N$, of spacing $c_{1}$ forming a circular lattice enclosing the A-B flux. The Schrödinger equation for this system reduces to the set of tight-binding equations [13]

$$
\begin{gathered}
-e^{i \frac{2 \pi}{N} \frac{\phi}{\phi_{0}}} \varphi_{n+1}-e^{-i \frac{2 \pi}{N} \frac{\phi}{\phi_{0}}} \varphi_{n-1}+\varepsilon_{n} \varphi_{n}=E \varphi_{n} \\
n=2,3, \ldots, N-1 \\
-e^{i \frac{2 \pi}{N} \frac{\phi}{\phi_{0}}} \varphi_{2}-e^{-i \frac{2 \pi}{N} \frac{\phi}{\phi_{0}}} \varphi_{N}+\varepsilon_{1} \varphi_{1}=E \varphi_{1} \\
-e^{i \frac{2 \pi}{N} \frac{\phi}{\phi_{0}}} \varphi_{1}-e^{-i \frac{2 \pi}{N} \frac{\phi}{\phi_{0}}} \varphi_{N-1}+\varepsilon_{N} \varphi_{N}=E \varphi_{N}
\end{gathered}
$$

where $\varphi_{n}$ denotes the amplitude of an eigenstate wavefunction at site $n, E$ and $\varepsilon_{m}(m=$ $1,2, \ldots N)$ are the corresponding eigenvalue and random site energies in units of a fixed nearest-neighbour hopping parameter. The flux-dependent phase factors in (1-3) (where $\varphi_{0}=h c / e$ is the flux quantum, with $h$ the Planck constant, $c$ the speed of light and $-e$ the electron charge) describe exactly [14] the effect of the flux-modified boundary conditions by which the effect of an A-B flux on the wave function of a tight-binding ring is generally introduced [1, 15]. 
The inverse localization length, $\xi^{-1}$, of states of energy $E$ of the ring threaded by the flux $\phi$ may be defined by $[9]$

$$
\begin{aligned}
\frac{1}{\xi} & =\lim _{n \rightarrow \infty} \frac{1}{n c_{1}}\left\langle\ln \varphi_{n}\right\rangle, \\
& =\lim _{n \leq N \rightarrow \infty} \frac{1}{n c_{1}} \sum_{p=2}^{n}\left\langle\ln \left|R_{p}\right|\right\rangle,
\end{aligned}
$$

where we have defined the amplitude ratios (Riccati variables) $R_{n}=\varphi_{n} / \varphi_{n-1}, n=2,3, \ldots N$ and $Q_{1}=\varphi_{1} / \varphi_{N}$ in terms of which (11-3) take a convenient form for recursive solution[10], starting from an arbitrary $\varphi_{1}$ at the initial site $n=1$. The angular brackets in (4) denote averaging over the disorder.

Detailed analytic solutions of Eqs. (1-3), rewritten in terms of the Riccati variables and for a non hermitian field $h$ substituted for $\frac{i 2 \pi \phi}{c_{1} \phi_{0}}$

$$
h \leftrightarrow \frac{i 2 \pi \phi}{c_{1} \phi_{0}}
$$

have been discussed previously [10] to second order in the random sites energies, for arbitraty magnitude of $h$. In Ref. 10 we performed the averaging of $\ln R_{n}$ expressed in terms of zeroth-, first- and second order contributions, $R_{n}^{(0)}, R_{n}^{(1)}$ and $R_{n}^{(2)}$, in $R_{n}$, namely

$$
\begin{aligned}
\ln \left|R_{n}\right|=\frac{1}{2}\left(e^{-i q} R_{n}^{(1)}+\text { c.c. }\right) & \\
& +\frac{1}{2}\left[e^{-i q}\left(R_{n}^{(2)}-\frac{e^{-i q}}{2} R_{n}^{(1) 2}\right)+c . c .\right], n=2,3, \ldots N,
\end{aligned}
$$

where

$$
R_{n}^{(0)}=e^{i q}
$$

is related to the energy by

$$
E=2 \cos \left(q+\frac{2 \pi}{N} \frac{\phi}{\phi_{0}}\right),
$$

(where eigenstate energies in the absence of disorder correspond to values $q=\frac{2 \pi}{N} k, k=$ $0,1,2 \ldots)$, assuming the site energies to be identically distributed idenpendent Gaussian variables: 


$$
\left\langle\varepsilon_{i}\right\rangle=0,\left\langle\varepsilon_{i} \varepsilon_{j}\right\rangle=\varepsilon_{0}^{2} \delta_{i, j}, i=1,2, \ldots, N
$$

We refer to[10] for the final explicit results for $\left\langle R_{n}^{(1) 2}\right\rangle$ and $\left\langle R_{n}^{(2)}\right\rangle$ (in the non-hermitian field case), using the identification(5). The inverse localization length (4) of the eigenstates of the ring threaded by the flux $\phi$ obtained from Eq (21) of Ref. 10 is given by

$$
\frac{1}{\xi}=-\frac{\varepsilon_{0}^{2}}{4} \frac{a}{(1-a)^{2}} \lim _{N \rightarrow \infty}\left(\frac{1+a^{N}}{1-a^{N}}\right)+\text { c.c. },
$$

where

$$
a=e^{-2 i\left(q+\frac{2 \pi}{N} \frac{\phi}{\phi_{0}}\right)} .
$$

The analysis of (10) in Ref. 10 for the case of an imaginary vector potential corresponding to the real field parameter $h$ leads to a finite so-called inverse directed localization length (IDLL) at energy $E$ (Eq. (31) in Ref. 10). In contrast, in the present case we obtain from (8),11)

$$
\begin{aligned}
\frac{1}{\xi} & =-i \frac{\varepsilon_{0}^{2}}{4} \frac{\cot \left(q N+\frac{2 \pi \phi}{\phi_{0}}\right)}{4-E^{2}}+c . c . \\
& =0
\end{aligned}
$$

which shows that the solutions of the Scrödinger equations (11-3) in the presence of an A-B flux are delocalized at order $\varepsilon_{0}^{2}$ i.e. we expect that $\frac{1}{\xi}=O\left(\varepsilon_{0}^{4}\right)$. Since this result is obtained for any $E$ we find, in particular, that the exact eigenstates of the ring threaded by an A-B flux have strongly enhanced localization lengths.

This contrasts with the exact result for the inverse localization length for weak disorder in a linear chain $(\phi=0)$ given by the celebrated Thouless formula 16, 17, 18, 19], namely

$$
\frac{1}{\xi_{1}}=\frac{\varepsilon_{0}^{2}}{2} \frac{1}{4-E^{2}}
$$

which is of order $\varepsilon_{0}^{2}$. We recall that (13) has also been rederived in Ref. 10, using Eq. (41). In this case the validity of the result is supported by the general theorems of Fürstenberg and of Oseledec[9] on the properties of infinite products of independent random $2 \times 2$ (transfer) matrices, in terms of which the inverse localization length of an unbounded linear chain 
may be defined. The localization length in a one-dimensional disordered ring at $\phi=0$ has sometimes been identified with the Thouless expression (13) for a linear chain (see e.g. [20]). However, no valid proof for this identification has been provided. As a result, there exists at present no analytic theory of localization on a weakly disordered one-dimensional ring at zero flux. In contrast, the present work based on Ref.[10] does provide a theory of localization for the case of a ring threaded by a finite A-B flux.

Our result (12) suggests, in particular, that persistent current in weakly disordered metallic rings threaded by an AB-flux is carried by states which are delocalized (i.e. free electronlike) to order $\varepsilon_{0}^{-2}$. The delocalized states (to the order $\varepsilon_{0}^{-2}$ ) induced by the AB-flux imply the existence of a quasi-metallic domain for ring perimeters lying between the elastic scattering mean free path, $\ell$, and the localization length, $\xi>>\ell$, at finite flux. In the absence of the flux such a domain does not exist since $\xi$ is then of the order of $\ell$ for a one-dimensional system[21].

Finally, we observe that in our recent study of persistent current in the tight-binding ring described by (1+3) [14], the effect of a weak disorder enters generally via an overall renormalization factor of the free electron current in the absence of disorder. Similar conclusions have also been reached in the study of persistent current in the more general case of spatially correlated random potentials [22]. In this sense our finding that the eigenstates of a weakly disordered ring threaded by an A-B flux are delocalized to order $\varepsilon_{0}^{-2}$ is consistent with our earlier results for persistent current [14, 22].

\section{REFERENCES}

[1] M. Büttiker, Y. Imry, and R. Landauer, Phys. Lett. 96A, 365 (1983).

[2] L.P. Lévy, G. Dolan, J. Dunsmuir and H. Bouchiat, Phys. Rev. Lett. 64, 2074 (1990).

[3] V. Chandraschkar; R.A. Webb, M.J. Brady, M.B. Ketchen, W.J. Gallagher, and A. Kleinsasser, Phys. Rev. Lett. 67, 3578 (1991).

[4] D. Mailly, C. Chapelier and A. Benoit, Phys. Rev. Lett. 70, 2020 (1993).

[5] E.M.Q. Jariwala, P. Mohanty, M.B. Ketchen, and R.A. Webb, Phys. Rev. Lett. 86, 1594 (2001).

[6] R. Deblock, R. Bel, B. Reulet, H. Bouchiat, and D. Mailly, Phys. Rev. Lett. 89, 206803 (2002). 
[7] Y. Imry, Introduction to Mesoscopic Physics (Oxford University Press, Oxford, 1997).

[8] K. Ishii, Prog. Theor. Phys., Suppl. 53, 77 (1973).

[9] A. Crisanti, G. Paladin and A. Vulpiani, Products of Random Matrices in Statistical Physics (Springer, Berlin, 1993).

[10] J. Heinrichs, Phys. Stat. Sol.(b) 231,19 (2002).

[11] J. Heinrichs, Pramana-J. Phys. 58, 153-171 (2002) (Proceedings of Int. Discussion Meeting on Mesoscopic and Disordered Systems, Bangalore, 18-20 December 2000, Ed. A.M. Jayannavar, H.R. Krishnamurthy and A.K. Raychariduri).

[12] N. Hatano and D.R. Nelson, Phys. Rev. Lett. 77, 570 (1996).

[13] H.F. Cheung, Y. Gefen, E.K. Riedel and W.H. Shih, Phys. Rev. B37, 6050 (1988).

[14] J. Heinrichs, Int. J. Mod. Phys. B16, 593 (2002).

[15] N. Byers and C.N. Yang, Phys. Rev. Lett. 7, 46 (1961).

[16] D.J. Thouless, in Ill-Condensed Matter, Edts R. Balian, R. Maynard and G. Toulouse, North Holland, Amsterdam, 1979.

[17] J.M. Luck, Les Systèmes Désordonnés Unidimensionnels, 1992 (Collection AléaSaclay)(Saclay:Commissariat de l'Energie Atomique).

[18] B. Kramer and A. MacKinon, Rep. Prog. Phys. 56, 1469 (1993).

[19] J. Pendry, Adv. Phys. 43, 461 (1994).

[20] P.W. Brouwer, P.G. Silvestrov, and W.C.J. Beenakker, Phys. Rev. B 56, R4333 (1997).

[21] D.J. Thouless, J. Phys. C6, 649 (1973).

[22] J. Heinrichs, J. Phys.: Condensed Matter 20, 245232 (2008). 